\title{
Firm mass in thyroid of an elderly patient: not always cancer
}

\author{
Alessandro Prete ${ }^{1, *}$, Giada Cosentino(D1,*, Luca Manetti², Carlo Enrico Ambrosini³ ${ }^{3}$ Piermarco Papini³, \\ Michele Marinò' ${ }^{1}$, Liborio Torregrossa ${ }^{4}$, Claudio Marcocci², Rossella Elisei² and \\ Isabella Lupi ${ }^{2} 2$ \\ 1 Unit of Endocrinology, University of Pisa, Pisa, Italy, 2 Unit of Endocrinology, Azienda Ospedaliero Universitaria \\ Pisana, ${ }^{3}$ Unit of Endocrine Surgery, Azienda Ospedaliero Universitaria Pisana, and ${ }^{4}$ Molecular Pathology and Critical \\ Area, Anatomic Pathologic Section, Azienda Ospedaliero Universitaria Pisana, Pisa, Toscana, Italy

\section{Summary}

In elderly patients presenting with a solid thyroid mass, the differential diagnosis between benign and malignant lesion is not always straightforward. We present the case of an 85-year-old woman with fever and an enlarged, firm and painful thyroid mass. Blood exams documented a mild thyrotoxicosis with a moderate inflammatory status. Thyroid scintiscan showed an absent uptake of 131I. Ultrasound and CT scan documented a $3 \mathrm{~cm}$ hypoechoic nodule with infiltration of the sternocleidomastoid muscle, very suspicious for neoplastic nature. Fine-needle aspiration and tru-cut biopsy were performed. During biopsy, the lesion was partially drained and a brownish fluid was extracted. The culture resulted positive for Klebsiella pneumoniae whereas the pathological analysis of the specimen was not conclusive due to the presence of an intense inflammatory response. A targeted oral antibiotic therapy was then initiated, obtaining only a partial response thus, in order to achieve a definite diagnosis, a minimally invasive hemithyroidectomy was performed. The pathological analysis documented acute suppurative thyroiditis and the clinical conditions of the patient significantly improved after surgical removal of thyroid abscess. In elderly patients with a solid thyroid mass, although neoplastic origin is quite frequent, acute suppurative thyroiditis should be considered as a differential diagnosis.

\section{Learning points:}

- A solid and rapidly growing thyroid mass in elderly patients can hide a multifaceted variety of diseases, both benign and malign.

- A multidisciplinary team (endocrinologist, surgeon, radiologist and pathologist) could be necessary in order to perform a correct differential diagnosis and therapeutic approach.

- Surgery can be decisive not only to clarify a clinically uncertain diagnosis, but also to rapidly improve the clinical conditions of the patient.

\section{Background}

Anterior neck swelling accompanied by pain could conceal a broad range of diseases, mainly of neoplastic or inflammatory/infective origin. The differential diagnosis between these two entities may be challenging for overlapping spectrum of signs and symptoms.
Of the malignancies, anaplastic thyroid cancer (ATC) accounts for $1-2 \%$ of all thyroid cancers and it is more frequent in the sixth and seventh decade of life with a female to male ratio of 1.5:2 (1). In almost all cases, patients with ATC present a firm thyroid mass rapidly expanding 
and usually doubling the volume within 1 week. This rapid growth causes an insufficient blood supply ensuing hemorrhage and local necrosis, thus facilitating a series of infections eventually leading to abscess formation. Patients usually complain for neck pain, pharyngodynia, asthenia and often fever. Among inflammatory-infective thyroid diseases, the most common cause of anterior neck pain in adults is subacute granulomatous thyroiditis (ST). ST is likely sustained by a viral infection, such as coronavirus (2); evidence for this etiology includes an antecedent history of upper respiratory tract infection, its self-limiting course and the seasonal distribution. A genetic predisposition could increase the risk for the occurrence of ST; indeed, an association between ST and HLA-Bw 35 has been reported in approximately two-thirds of Caucasians and Chinese patients (3).

Acute suppurative thyroiditis (AST) is an even more uncommon inflammatory condition representing $0.1-0.7 \%$ of all thyroid diseases. In almost all cases, AST results from a bacterial infection, although a wide spectrum of microbial pathogens is reported in literature ranging from fungi to parasitic organisms, especially in immunocompromised patients (4). Pyriform sinus fistulae, which are mostly seen in children, are considered to be the most common route of the thyroidal infection and the left thyroid lobe, due to anatomic conformation, is more frequently involved. Infection may reach the thyroid gland through several paths including penetrating injuries, hematogenous spread from distant sites, or via the lymphatics as a result of local infections such as pharyngitis or mastoiditis. Interestingly, a pre-existing thyroid disease, most commonly nodular goiter, has been reported to be present in more than $50 \%$ of cases (4).

Laboratory findings, such as leukocytosis with elevated $\mathrm{C}$ reactive protein (CRP) and erythrocyte sedimentation rate (ESR) are not specific to discriminate ST from AST. Conversely, thyroid function tests and ultrasound pattern are more helpful in the differential diagnosis between these two forms. ST is associated with a triphasic clinical course that progresses from thyrotoxicosis to hypothyroidism and eventually to restoration of normal function. At the end of the inflammatory process, a persistent hypothyroidism may develop in $10 \%$ of the cases (5). In AST, euthyroidism is the most common hormonal feature although transient elevations in serum thyroxine levels have also been reported. As in the case of ST, the thyroid follicle damage due to the inflammatory process may lead to a discharge of preformed thyroid hormone thus manifesting with thyrotoxicosis. Due to its destructive pathogenesis, in both ST and AST, thyrotoxicosis is characterized by low radioiodine uptake (3). Whereas ST is self-limiting and often requires glucocorticoid to speed the process and alleviate the pain, long-term management of AST primarily focuses on antibiotic therapy although an invasive approach such as abscess drainage or surgery may be necessary in few settings (3). Finally, in particular, when the thyroid mass is extremely hard or clinical history is positive for other autoimmune disorders, Riedel's thyroiditis (RT) should be also excluded. RT is an invasive fibrous thyroiditis which involves the thyroid parenchyma and surrounding tissue structures (6). This disorder is the rarest among all thyroiditis, it affects primarily women (female-to-male ratio of 3:1) with an age range of 30-60 years and, recently, it has been included among the manifestations of IgG4-related disease.

\section{Case presentation}

An 85-year-old woman presented to our Endocrine Unit with a hard and aching right-side neck mass without any other signs of local inflammation. Ten days before, she had started complaining of pharyngodynia and fever $\left(38^{\circ} \mathrm{C}\right)$ that was treated with antibiotics without obtaining a satisfying clinical response.

Her clinical history was unremarkable except for thyroid nodule and bullous pemphigoid that was treated with topical glucocorticoids few years before.

\section{Investigation}

At admission, her blood tests revealed a moderate thyrotoxicosis without TPOAb, TgAb and TSH receptor antibodies, an inflammatory state with severely elevated CRP, moderate elevation of fibrinogen, and high levels of total and fractionated $\mathrm{IgG}$, and low levels of procalcitonin (PCT) (Tables 1 and 2).

To better characterize the thyrotoxicosis, a thyroid 131-I scintiscan was performed, revealing a low uptake of $131 \mathrm{I}$ at $3 \mathrm{rd}(2.8 \%)$ and 24 th hour $(2.3 \%)$ as shown in Fig. 1A. Urinary iodine excretion was in the normal range. The ultrasound examination documented an irregular hypoechoic $7 \mathrm{~cm}$ nodule of the right thyroid lobe with micro- and macro-calcifications, invading the sternocleidomastoid muscle and with multiple enlarged neck lymph nodes (Fig. 1B). The CT scan confirmed the infiltration of the cervical muscles and revealed tracheal and vasal compression, thus these signs were considered highly suspicious for malignancy (Fig. 1D). 
Table 1 Laboratory investigations of thyroid function at baseline, after antibiotics therapy and after abscess excision.

\begin{tabular}{|c|c|c|c|c|}
\hline & $\begin{array}{c}\text { At } \\
\text { baseline }\end{array}$ & $\begin{array}{c}\text { After } 2 \\
\text { weeks of } \\
\text { antibiotics } \\
\text { therapy }\end{array}$ & $\begin{array}{c}\text { After } 1 \\
\text { week } \\
\text { from } \\
\text { abscess } \\
\text { excision }\end{array}$ & $\begin{array}{l}\text { Reference } \\
\text { range }\end{array}$ \\
\hline TSH (mUI/L) & 0.018 & 0.332 & 2.970 & $0.400-4000$ \\
\hline fT3 (ng/L) & 4.84 & 3.19 & 2.91 & $2.70-5.70$ \\
\hline fT4 (ng/dL) & 2.63 & 1.15 & 0.90 & $0.70-1.70$ \\
\hline $\operatorname{AbTg}(U \mathrm{I} / \mathrm{mL})$ & 5 & $<1.0$ & 17 & 0-30 \\
\hline $\begin{array}{l}\text { AbTPO } \\
\text { (UI/mL) }\end{array}$ & $<1.0$ & $<1.0$ & $<1.0$ & $0-10$ \\
\hline TRAB (UI/L) & $<0.04$ & $<0.04$ & - & $0-1.00$ \\
\hline $\operatorname{Tg}(\mu g / L)$ & 395.93 & 34.58 & 15.34 & $0-35$ \\
\hline
\end{tabular}

Esophagogastroduodenoscopy and tracheobroncoscopy resulted negative for any neoplastic infiltration. Trucut biopsy was performed: pathology revealed an intense inflammatory process with granulation tissue, abundant neutrophils, and not-clearly benign interspersed spindle cells with immunophenotype suggesting an endothelial differentiation. A drainage of the nodule was also performed and purulent fluid was extracted. The cultural exam and antibiogram of the specimen resulted positive for Klebsiella pneumoniae, sensitive to amoxicillin-clavulanic acid.

\section{Treatment}

Targeted-antibiotic therapy with 2 g per days of amoxicillina-clavulanic acid was started, however, since a significant reduction of the lesion was not documented at ultrasound (Fig. 1B and C), and the pathology examination on tru-cut was not able to exclude with certainty the neoplastic origin, we decided to perform

Table 2 Laboratory investigations to assess the inflammatory status before and after abscess excision.

\begin{tabular}{|c|c|c|c|}
\hline & $\begin{array}{c}\text { Before } \\
\text { abscess } \\
\text { excision }\end{array}$ & $\begin{array}{l}\text { After abscess } \\
\text { excision }\end{array}$ & Reference range \\
\hline $\operatorname{lgA}(\mathrm{mg} / \mathrm{dL})$ & 458 & 348 & $70-400$ \\
\hline IgD (U/mL) & 25 & 29 & $0-100$ \\
\hline IgE (U/mL) & 1060 & 752 & $50-120$ \\
\hline $\operatorname{lgG}(\mathrm{mg} / \mathrm{dL})$ & 1790 & 1310 & $700-1600$ \\
\hline $\operatorname{lgG} 1(\mathrm{mg} / \mathrm{dL})$ & 1200 & 790 & $545-735$ \\
\hline $\operatorname{lgG2}(\mathrm{mg} / \mathrm{dL})$ & 536 & 350 & 290-395 \\
\hline $\operatorname{lgG3}(\mathrm{mg} / \mathrm{dL})$ & 56 & 121 & $37-50$ \\
\hline $\operatorname{lgG} 4(\mathrm{mg} / \mathrm{dL})$ & 72 & 49 & $49-66$ \\
\hline $\operatorname{lgM}(\mathrm{mg} / \mathrm{dL})$ & 76 & 65 & $40-230$ \\
\hline CRP (mg/dL) & 10.95 & 1.09 & 0,5 \\
\hline $\begin{array}{l}\text { WBC count } \\
\left(10^{3} / \mu \mathrm{L}\right)\end{array}$ & 5.66 & 4.87 & $4-11$ \\
\hline РСТ (ng/mL) & 0.17 & 0.05 & 0.5 \\
\hline
\end{tabular}
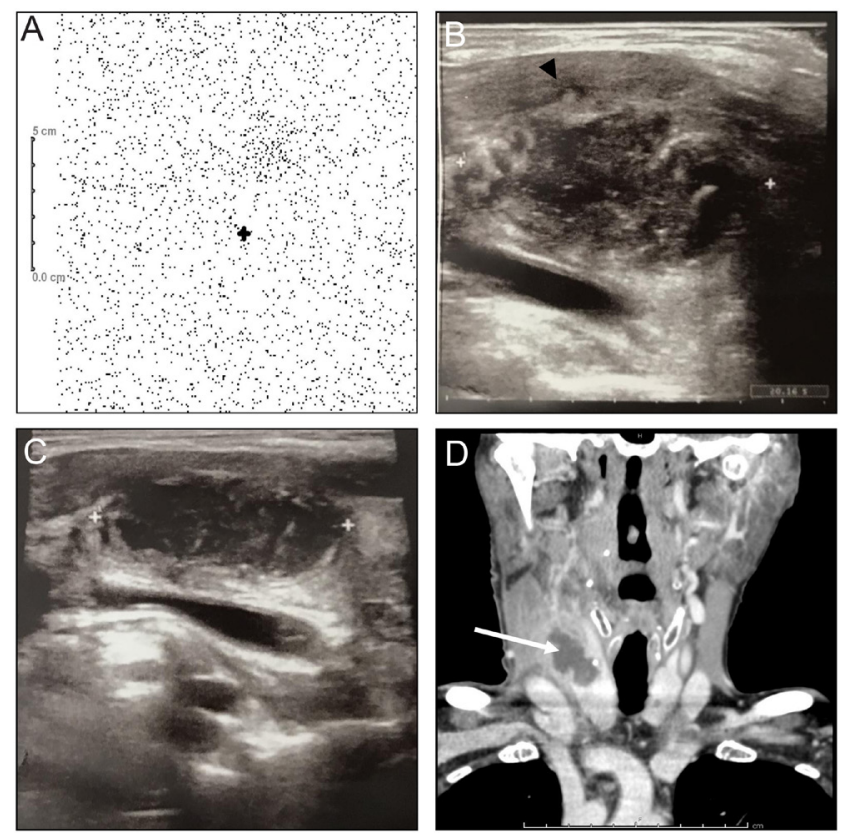

Figure 1

(A) 131-I scintigraphy scan described low uptake of 131-radioiodine at 3rd and 24th hour (the cross identifies jugular region). (B) US scan documented $3 \mathrm{~cm}$ nodular lesion with sternocleidomastoid muscle infiltration, which is highlighted by a black triangle. (C) US scan, performed after 10 days of antibiotic therapy, documented only a partial reduction after antibiotic therapy. (D) CT scan documented an irregular nodular lesion, with peripheral vascular tissue and wide necrotic nucleus (white arrow), infiltrating sternocleidomastoid muscle.

an hemithyroidectomy. The histological examination excluded malignancy and showed a variegated architecture: a central area of colliquative necrosis delimited by florid granulation tissue with abundant fibroblasts and fibrosis, and intense acute and chronic inflammatory infiltrate with numerous macrophages and plasma cells. Infiltrating macrophages appeared both as histiocytes with hemosiderinic pigment and as 'foreign body'-type giant cells around cholesterol crystals. There was no evidence of multinucleated giant cells with the formation of granulomas as well as occlusive phlebitis. Moreover, immunohistochemistry for IgG4 staining was negative. The inflammatory process seemed to incorporate residual thyroid nodule composed of microfollicles surrounded by thick fibrous capsule and showed evident spread to soft and muscular peri-thyroidal tissues (Fig. 2).

\section{Outcome}

Biochemical and hormonal tests, performed after 7 and 28 days, documented a euthyroid state and a substantial remission of the inflammatory state (Tables 1 and 2). 

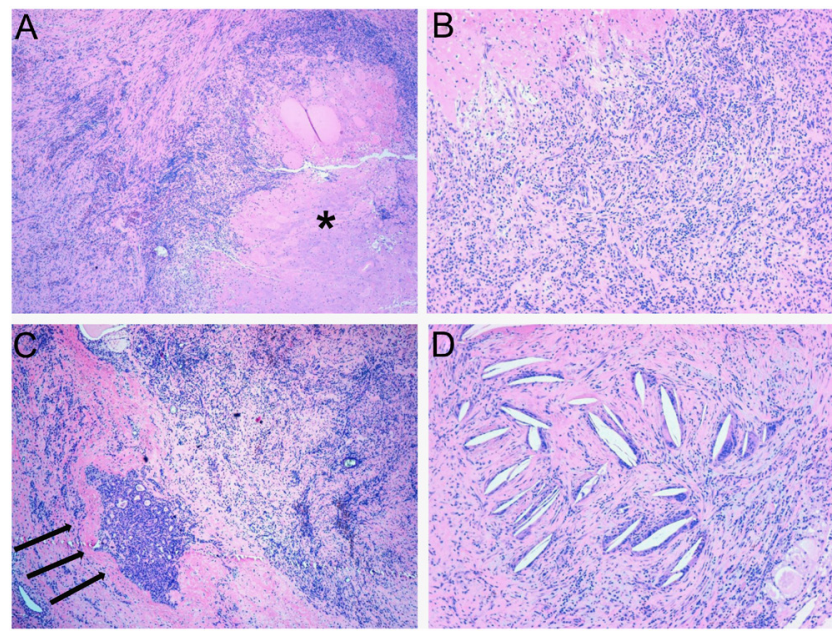

Figure 2

Histological representative pictures (hematoxylin and eosin). (A) Central area of colliquative necrosis (*Colliquative necrosis; original magnification $4 \times$ ). (B) Granulation tissue adjacent to colliquative necrosis (original magnification 10x). (C) Marginal and residual thyroid nodule composed of microfollicles surrounded by thick fibrous capsule (see arrows; original magnification 4×). (D) Detail of macrophages appearing as 'foreign body'-type giant cells around cholesterol crystals in a context of marked fibrosis (original magnification 10x).

\section{Discussion}

We report the case of an elderly patient who presented with a hard-thyroid nodule that rapidly grew in a few weeks. She presented symptoms such as pharyngodynia and moderate fever, which are typically associated with inflammatory thyroid diseases. However, the differential diagnosis between aggressive thyroid cancer and benign inflammatory disease was mandatory, because symptoms as dysphagia, neck pain, dysphonia can accompany the enlargement of a firm neck mass in both cases $(4,7)$. On one hand, necrosis pattern and abscess formation may be present in aggressive cancers (1) and, on the other hand, abscess formation may evade thyroid bed and infiltrate adjacent tissues, simulating aggressive neoplasm behavior (4). Moreover, both of them are usually described as a 'cold' nodule at thyroid scintigraphy $(1,4)$. Fine needle aspiration or trucut biopsy are usually valuable tools to obtain a definite diagnosis and culture of its specimen is crucial to determine infective etiology and to guide its treatment (4). However, in our case, the material obtained from FNAC and trucut biopsy were inconclusive to rule out a thyroid malignancy due to the presence of spindle cells that resembled aggressive cancer cells.

AST may include a various spectrum of clinical pictures (8) and is a potentially life-threatening endocrine disease (3). Its treatment comprises two levels of management. The first level comprises US-FNA drainage that is useful in order to evacuate the abscess and to guide the antimicrobial therapy; the second level consists of long-term treatment that depends on the persistence of the abscess and presence of piriform sinus tract fistula (3). In our case, in spite of antibiogram guided therapy, the abscess was persisting. Hemithyroidectomy was considered necessary for a diagnosis of certainty, and to treat the infectious state (Table 3). Accordingly, after excision of the abscess, the patient presented a decrease of CRP, PCT and total IgG and a general improvement of clinical conditions was achieved.

The increase of CRP is present also in ST, which we also considered in the differential diagnosis. Moreover, the clinical history of pharyngodynia and symptoms (as pain and moderate fever) supported this diagnosis as well as the thyrotoxic status (first stage of the classical triphasic trend of thyroid function in ST) (Table 1) (5). Furthermore, the scintigraphy scan was consistent with a destructive process involving the entire thyroid gland. However, US scan and pathology findings did not confirm this suspect: the former did not identify a diffuse heterogeneity of the gland and the latter showed an intense acute and chronic inflammatory pattern without any hallmark of ST such as multinucleate giant cells or granulomas (5). Accordingly, subacute thyroiditis diagnosis was ruled out.

Patient's history, positive for autoimmune disease (bullous pemphigoid) led us to also rule out RT diagnosis. RT criteria for diagnosis include: (a) presence of fibroinflammatory lesions, (b) extension into the adjacent tissues, involving strap muscles, (c) infiltration of inflammatory cells without giant cells, lymphoid follicles, oncocytes or granulomas, (d) presence of occlusive phlebitis and (e) absence of cancer (6). Moreover, recent evidences included RT as part of a more complex spectrum of manifestations recognized as IgG4-related disease (9). IgG4-related disease is a fibroinflammatory condition presenting with tumefactive lesions, dense lymphoplasmacytic infiltrate rich in IgG4-positive plasma cells and fibrosis that can be virtually described in every organ. It can often, but not always, present elevated serum IgG4 concentrations (9). In our case, there was a mild increase of IgG4 in the context of an elevation of total IgG, consistent with an inflammatory response to an infectious agent. Moreover, in spite of the presence of fibroinflammatory response with inflammatory cells and the extension of the lesion into the adjacent tissues, IgG4 immunohistochemistry for IgG4 was negative and occlusive phlebitis was not present. Therefore, 
Table 3 Clinical features of acute suppurative, subacute and Riedel thyroiditis.

\begin{tabular}{|c|c|}
\hline & Acute suppurative thyroiditis \\
\hline Incidence & $0.1-0.7 \%$ of all thyroid disease \\
\hline Etiological cause & $\begin{array}{l}\text { Infectious (usually associated } \\
\text { with piriform sinus fistula), } \\
\text { radiation and traumatic }\end{array}$ \\
\hline Symptoms & $\begin{array}{l}\text { Anterior neck swelling, pain } \\
\text { and fever }\end{array}$ \\
\hline $\begin{array}{l}\text { Inflammation } \\
\text { markers }\end{array}$ & $\begin{array}{l}\text { Increase in WBC, PCT, ESR and } \\
\text { CRP }\end{array}$ \\
\hline Thyroid function & $\begin{array}{l}\text { Usually euthyroid state, rare } \\
\text { thyrotoxicosis due to follicle } \\
\text { destruction }\end{array}$ \\
\hline Ultrasound & $\begin{array}{l}\text { Hypoechoic area with } \\
\text { perithyroidal hypoechoic } \\
\text { space }\end{array}$ \\
\hline $\begin{array}{l}\text { Scintigraphy scan } \\
(131-I)\end{array}$ & $\begin{array}{l}\text { In presence of thyrotoxicosis } \\
\text { state: reduced/absent tracer } \\
\text { uptake }\end{array}$ \\
\hline $\begin{array}{l}\text { Histological } \\
\text { features }\end{array}$ & Abscess \\
\hline Treatment & $\begin{array}{l}\text { Percutaneous or surgical } \\
\text { drainage, antibiotic therapy } \\
\text { and fistula obliteration }\end{array}$ \\
\hline Long term outcome & Usually excellent prognosis \\
\hline
\end{tabular}

Riedel thyroiditis

1.06 per 100000 person/year

Unclear, probably local manifestation

of IgG4 related disease

Firm mass in thyroid with compressive symptoms

Normal/moderately elevated WBC

and ESR

Frequent hypothyroidism state

(Thyrotoxicosis, hypothyroidism and euthyroidism)

Diffuse heterogeneity and low intensity vascular flow

Hypoechoic and low intensity vascular flow

Heterogeneous and typically low tracer uptake

absent tracer uptake;

Recovery phase: irregular or diffusely increased tracer uptake

Multinucleate giant cells and mononuclear cells infiltration

(a) Fibroinflammatory of all or a portion of the thyroid:

(b) Extension into the adjacent tissues, including strap muscles;

(c) Infiltration of inflammatory cells without giant cells, lymphoid follicles, oncocytes or granulomas;

(d) Presence of occlusive phlebitis

(e) Absence of cancer

Glucorticoids or nonsteroidal

Glucorticoids, tamoxifen or surgery

anti-inflammatory drugs

Possible permanent

hypothyroidism;

Frequent hypothyroidism

Possible recurrence (1.4-4\%)

the anatomopathological analysis was not compatible with RT.

\section{Conclusions}

In conclusion, even if thyroid cancer is more frequent in the elderly with a firm neck mass, we should exclude a benign disorder such as acute suppurative thyroiditis which is quite rare. To date, no clinical or imaging hallmarks for diagnosing the nature of the lesion could be considered singularly, although a full evaluation of the patient is necessary. Awareness of the heterogeneity in the presentation of neck masses is crucial to guide endocrinologists in daily clinical practice.

\section{Declaration of interest}

The authors declare that there is no conflict of interest that could be perceived as prejudicing the impartiality of the research reported.

\section{Funding}

This research did not receive any specific grant from any funding agency in the profit, commercial or not-for-profit sector.

\section{References}

1 Molinaro E, Romei C, Biagini A, Sabini E, Agate L, Mazzeo S, Materazzi G, Sellari-Franceschini S, Ribechini A, Torregrossa L, et al. Anaplastic thyroid carcinoma: from clinicopathology to genetics and advanced therapies. Nature Reviews: Endocrinology 201713 644-660. (https://doi.org/10.1038/nrendo.2017.76)

2 Brancatella A, Ricci D, Viola N, Sgrò D, Santini F \& Latrofa F. Subacute thyroiditis after Sars-COV-2 infection. Journal of Clinical Endocrinology and Metabolism 2020105 dgaa276. (https://doi. org/10.1210/clinem/dgaa276)

3 Paes JE, Burman KD, Cohen J, Franklyn J, McHenry CR, Shoham S \& Kloos RT. Acute bacterial suppurative thyroiditis: a clinical review and expert opinion. Thyroid 201020 247-255. (https://doi. org/10.1089/thy.2008.0146)

4 Al-Dajani N \& Wootton SH. Cervical lymphadenitis, suppurative parotitis, thyroiditis, and infected cysts. Infectious Disease Clinics of North America 200721 523-541, viii. (https://doi.org/10.1016/j. idc.2007.03.004) 
Endocrinology

Diabetes \& Metabolism

CASE REPORTS
A Prete, G Cosentino and

others
5 Benbassat CA, Olchovsky D, Tsvetov G \& Shimon I. Subacute thyroiditis: clinical characteristics and treatment outcome in fifty-six consecutive patients diagnosed between 1999 and 2005. Journal of Endocrinological Investigation 200730 631-635. (https://doi. org/10.1007/BF03347442)

6 Kottahachchi D \& Topliss DJ. Immunoglobulin G4-related thyroid diseases. European Thyroid Journal 20165 231-239. (https://doi. org/10.1159/000452623)

7 Tollefsen HR, DeCosse JJ \& Hutter RV. Papillary carcinoma of the thyroid, A clinical and patholohical study of 70 fatal cases.
Cancer 196417 1035-1044. (https://doi.org/10.1002/10970142(196408)17:8<1035::aid-cncr2820170810>3.0.co;2-w)

8 Falhammar H, Wallin G \& Calissendorff J. Acute suppurative thyroiditis with thyroid abscess in adults: clinical presentation, treatment and outcomes. BMC Endocrine Disorders 201919 130. (https://doi.org/10.1186/s12902019-0458-0)

9 Stone JH, Zen Y \& Deshpande V. VIgG4-related disease. New England Journal of Medicine 2012366 539-551. (https://doi.org/10.1056/ NEJMra1104650)

Received in final form 24 September 2020

Accepted 8 October 2020 